\title{
Avanços no Diagnóstico e Diagnóstico Diferencial das Epilepsias"
}

Carlos José Reis de Campos ", Mauro Muszkat* , Roseli Mara Reis de Campos , José Geraldo de Camargo Lima

\section{RESUMO}

São apresentados recentes mélodos utilizáveis no diagnóstico e diagnóstico diferencial das epilepsias, especialmente métodos de neuro-imagem, estruturais (TC e RNM) e funcionais (PET e SPECT), bem como recentes métodos eletrofisiológicos como "Brain Mapping" e monitorizaçāo eletrencefalográfica ambulatorial prolongada e de video-EEG.

\section{UNITERMOS}

Epilepsias, TC, RNM, PET, SPECT, "Brain Mapping", monitorização eletrencefalográfica ambulatorial prolongada.
- Professor Titular; Chefe da Disciplina de Neurologia da Escola Paulista de Medicina.

- Professor Adjunto-Doutor da Disciplina de Neurologia da Escola Paulista de Medicina.

.. Doutor em Neurologia pela Escola Paulista de Medicina.

**** Pós-graduanda em Medicina Nuclear da Escola Paulista de Medicina
Além dos dados anamnésticos, de fundamental importância no diagnóstico e diagnóstico diferencial das epilepsias, passamos a contar nos últimos anos com modernos exames subsidiários nessa tarefa.

\section{Aplicação Diagnóstica da Neuro-Imagem em Epilepsias}

Com finalidades de tratamento cirúrgico de foco epileptogênico, é necessária a sua localização adequada, pois podem ser executadas ressecções mais limitadas como a das regiões amígdalo-hipocampal e neocortical temporal anterior. Para isso utilizam-se estudos de neuro-imagem cerebral, através de Tomografia Computadorizada (TC) e Ressonância Nuclear Magnética (RNM) para a detecção de alterações anatômicas e Ressonância Nuclear Magnética Espectral, Tomografia Computadorizada por Emissão de Fóton Único ("Single Photon Emission Computed Tomography" - SPECT) e Tomografia por Emissão de Pósitron ("Positron Emission Tomography" - PET) para alterações funcionais (Engel et al., 1992).

\section{Estudos Anatômicos}

A TC e RNM cerebrais possibilitam a identificação de neoplasias, infartos, hemorragias intracerebrais e doenças inflamatórias, mas nas epilepsias o achado patológico mais freqüente é a esclerose temporal mesial, que pode ser conseqüência de uma lesão prévia, apresentando-se agora como uma cicatriz gliótica. A esclerose hipócampal é a mais comum das lesões temporais meslaịs, entretanto, outras entidades como hamartomas, tumores gliais e malformaçðes vasculares podem ocorrer (Devous et al., 1990; Engel et al., 1992). A visualização da atrofia do hipocampo e/ou da amígdala, ou de outras lesões estruturais, associadas ou não a um sinal hiperintenso em imagens "T2 ponderadas" na RNM está relacionada a alterações patológicas, características da esclerose temporal mesial e fornecem dados para a lateralização do foco epiléptico. Outro estudo auxiliar na detecção da esclerose temporal mesial é a avaliação computadorizada pela RNM dos volumes da amígdala e hipocampo, fornecendo dados 
quantitativos que mostrarão uma redução volumétrica da área alterada (Jack et al., 1990; Cendes et al., 1993).

Em geral, a TC é a técnica de neuro-imagem menos sensível para a detecção de anormalidades em pacientes com crises epilépticas, muito embora tenha especial importância no diagnóstico das calcificações intracranianas da neurocisticercose, que constitui uma das etiologias mais freqüentemente encontradas para as epilepsias no Brasil.

\section{Estudos Funcionais}

Como as epilepsias constituem anormalidades da função cerebral, nem sempre são encontradas alterações anatômicas relacionadas com o foco epileptogênico, diagnosticado por critérios clínico-eletrencefalográficos ou através de imagem funcional (Devous et al., 1990).

A imagem cerebral funcional é atualmente uma técnica não-invasiva, bem estabelecida no manejo das epilepsias e, com sua maior resolução espacial, pode ser efetiva na localização do foco epiléptico, levando a uma importante contribuição diagnóstica, por fornecer elementos que auxiliam no tratamento cirúrgico de muitos pacientes com dificuldades de controle medicamentoso, diminuindo, com isto, o risco de estudos invasivos com finalidade de diagnóstico funcional e de controle evolutivo (Devous et al., 1990).

Anormalidades no fluxo sangüíneo cerebral regional e alterações metabólicas estão presentes em pacientes com epilepsias de difícil controle. Quando é considerada a ressecção cirúrgica em pacientes com epilepsia parcial, as imagens correspondentes à função cerebral regional anormal são de grande valor diagnóstico na localização da lesão epileptogênica (Bercovic et al., 1991).

A Tomografia por Emissão de Pósitron (PET) utiliza radioisótopos de meia-vida curta e que, portanto, devem ser produzidos em um ciclotron localizado próximo ao equipamento de PET. Os radioisótopos emissores de pósitron que são comumente incorporados aos traçadores ativos biologicamente para os estudos de PET e suas vidas médias incluem: Oxigênio-15 (123 segundos), Nitrogênio- 13 (10 minutos), Carbono-11 (20 minutos) e Flúor-18 (110 minutos). Esses elementos podem ser usados para marcar uma grande variedade de substâncias ou drogas sem alterar suas propriedades bioquímicas. Esses compostos marcados permitem uma medida do fluxo sangüíneo regional, da oxigenação cerebral e metabolismo de glicose, aminoácidos, síntese protéica, ligação de receptores e farmacocinética de drogas específicas. A maioria dos estudos de PET para epilepsia tem sido realizado usando o ${ }^{18} \mathrm{~F}$-fluordeoxiglicose (FDG). Entretanto, o alto custo do equipamento e dos profissionais necessários para a realização dos estudos de PET limitam o seu uso clínico (Engel, 1986).

Devido às dificuldades apontadas no uso do PET, tem sido utilizado, com maior frequiência, o estudo com SPECT cerebral para avaliação diagnóstica das epilepsias. A imagem de SPECT cerebral utiliza matrizes reais de até $128 \mathrm{X}$ 128 pixels e, mais recentemente, de $256 \times 256$ pixels, o que proporciona maior resolução espacial não interpolada em relação ao Mapeamento Eletrencefalográfico ("Brain Mapping"). O SPECT necessita de radiotraçadores que atravessem a barreira hematoencefálica, distribuam-se proporcionalmente ao fluxo sangüíneo cerebral regional e permaneçam fixados no cérebro por um tempo suficientemente longo para permitir a aquisição do exame (Nakamura et al., 1989). O fluxo sanguíneo cerebral pode ser medido quantitativamente através do gás inerte Xenônio-133, de vida-média muito curta, durante seu rápido clearance pelo cérebro. Porém, na atualidade, o rádio-fármaco de escolha é o $99 \mathrm{mTc}$-hexametilprolilenoaminoxima (HMPAO) que atravessa a barreira hematoencefálica numa relação proporcional ao fluxo sangüíneo cerebral e mostra uma prolongada retenção cerebral, permitindo em algumas situações o estudo seriado do paciente durante e após uma crise epiléptica (Holman \& Devous, 1992).

Em ambos os estudos funcionais de imagens cerebrais obtidas com SPECT e PET em pacientes epilépticos, observa-se na fase crítica (ictal) um aumento de captação do radioțraçador na região do foco epileptogênico e diminuição na fase intercrítica (interictal). Geralmente, a área correspondente às alterações encontradas nesses estudos mostrase de maior extensão em relação às imagens anatômicas da TC e RNM (Devous et al., 1990).

Quando se considera a lobectomia temporal em pacientes epilépticos, outra avaliação importante é a localização dos centros de memória, o que pode ser feito através do Teste de Wada, o qual consiste na administração intracarotídea de amital sódico para anestesiar um hemisfério cerebral, com realização simultânea de registro eletrencefalográfico contínuo e testes de memória, com finalidade de avaliação do desempenho do paciente, com apenas um hemisfério cerebral funcionante.

Prossegue-se com arteriografias seriadas, averiguandose a distribuição do amital sódico. Se o paciente mantiver sua memória inalterada com presença do contraste em um hemisfério cerebral, conclui-se que o centro de memória se localiza no outro hemisfério (Rausch \& Langfitt, 1991). Entretanto, como a arteriografia pode não refletir acuradamente a distribuição do amital sódico, superestimando-a, atualmente recomenda-se a utilização do amital combinado ao HMPAO, o que proporciona uma melhor determinação 
de sua distribuição intracerebral, com visualização da concentração do HMPAO no hemisfério cerebral estudado e ausência no contralateral. A interpretação errônea destes testes, sem dúvida, implica em riscos elevados de remoção de uma porção do lobo temporal, que pode conter centros da memória se apenas as arteriografias forem usadas como referências para acompanhar a distribuição do amital (Jeffrey et al., 1991).

A Ressonância Nuclear Magnética Espectral pode ser usada para avaliação metabólica através da medida dinâmica da função cerebral, por detecção de fosfato orgânico e pH intracelular durante crises epiléptcas (Brady \& Pykett, 1983; Petroff et al., 1984).

\section{Eletrencefalografia Computadorizada e "Brain Mapping"}

Os modernos sistemas de eletrencefalografia computadorizada (Gotman, 1985; Jacobs et al., 1991) e a melhoria da qualidade de impressão (Mainwaring et al., 1991) dos registros vêm fazendo com que eșses recursos sejam mais utilizados, acrescentando, em comparação com a eletrencefalografia convencional analógica, maiores possibilidades diagnósticas de grafo-elementos de difícil interpretação, através de modificações de ganho, com ampliação do sinal, e de velocidade de registro, bem como análise dessas figuras gráficas em diferentes montagens.

A utilização de procedimentos neurofisiológicos quantitativos utilizando-se microcomputadores tem aberto um novo e fértil campo de pesquisa em epileptologia clínica. $O$ mapeamento eletrencefalográfico computadorizado (MEC)-Brain Mapping, através da recomposição matemática dos ritmos elétricos cerebrais, permite maior precisão e objetividade na delimitação das mudanças nos padrões espaciais e temporais da atividade paroxística e da atividade elétrica cerebral de base (Lombroso \& Duffy, 1982; Miyauchi et al., 1991; Maurer \& Dierks, 1991).

O MEC pode contribuir para o diagnóstico e prognóstico das epilepsias em várias situações clínicas:

1) Identificação dos chamados "dipolos tangenciais", característicos das epilepsias parciais benignas (Panet \& Gotman, 1990, Gregory \& Wong, 1992).

2) Delimitação de paroxismos lentos e rápidos de baixa amplitude durante período intercrítico em EEGs convencionais considerados normais (Hughes et al., 1991; Panet \& Gotman, 1990).

3) Delimitação topográfica de focos primários e de projeção de descargas focais, uma vez que o padrão de distribuição das frequiências e amplitudes pode ser mensurado com precisão de milissegundos (Gregory \& Wong, 1992; Hughes et al., 1991; Panet \& Gotman, 1990). Assim, o MEC apresenta maior resolução temporal que o SPECT cerebral, mesmo utilizando apenas 20 canais para a efetiva captação dos sinais.

As vantagens do MEC sobre o EEG convencional não se baseiam somente nos estudos quantitativos dos ritmos cerebrais, mas na possibilidade de análise de correlativos funcionais de atividade mental complexa durante estimulação sensorial e cognitiva, com estudo das mudanças lateralizadas dos ritmos elétricos cerebrais durante provas cognitivas (Brown \& Lehmann, 1979; Petsche \& Pockberger, 1986; Freeman \& Maurer, 1989), estimulação auditiva verbal (teste dicótico) e não-verbal (musical) - (Breitiling et al., 1987). Esses métodos mais recentes de estudo poderão contribuir não só para delimitar, por meios não-invasivos, o hemisfério dominante para a linguagem em epilépticos, mas também para o estudo neuropsicológico funcional das estruturas amígadalo-hipocampais envolvidas nas descargas paroxísticas lateralizadas.

\section{Monitorização com Sistemas de Telemetria Vídeo-Eletrencefalográficos}

Para um preciso diagnóstico e localização de focos epilépticos pode ser necessária monitorização vídeo-eletrencefalográfica (Vuong \& Burgen, 1991). Como as crises epilépticas não são previsíveis, os pacientes devem ser monitorados de modo contínuo e prolongado por vários dias em unidades especialmente instaladas para esse fim. A monitorização combinada vídeo-EEG permite cuidadosa revisão simultânea das manifestações clínicas e eletrencefalográficas, com eletrodos de couro cabeludo e eletrodos especiais (esfenoidais, subdurais e de profundidade). Esta técnica, especialmente com métodos invasivos de colocação de eletrodos, é que tem propiciado diferenciar com precisão a origem das descargas epilépticas, frontal ou temporal, direita ou esquerda, definindo, assim, a área de abordagem cirúrgica para o tratamento de pacientes epilépticos refratários ao tratamento clínico.

\section{Monitorização Eletrencefalográfica Ambulatorial Prolongada (MEAP)}

Muitos pacientes que necessitam monitorização eletrencefalográfica não requerem hospitalização pela sua condição médica, a menos que tenham eletrodos invasivos ou necessitem a retirada de suas medicações anti-epilépticas. 
Eles são hospitalizados porque é onde o equipamento de monitorização está localizado. A monitorização no hospital com sistemas de telemetria com vídeo-EEg (Vuong \& Burgen, 1991) dobra, em média, o custo do procedimento (Ives et al., 1993) e é comum que muitos pacientes de difícil controle apresentem redução do número de crises ao serem hospitalizados (Riley et al., 1981). A monitorização eletrencefalográfica ambulatorial (MEAP) foi usada pela primeira vez há cerca de 20 anos atrás (Ives \& Woods, 1975).

A investigação de crises epilépticas tem sido a maior aplicação da monitorização eletrencefalográfica ambulatorial prolongada, já que esse exame prolongado permite o registro de distúrbios transitórios da função cerebral, auxiliando na classificação das crises epilépticas, diagnóstico diferencial das mesmas, avaliação da resposta ao tratamento e o estabelecimento da influência de fatores psicológicos, ou outros, no desencadeamento de crises (Ebersole, 1988, 1993).

Comparada com os procedimentos de registro eletrencefalográfico convencional, a monitorização ambulatorial prolongada tem a vantagem considerável de permitir, com custo financeiro relativamente baixo, o registro contínuo de sinais eletrofisiológicos por longos períodos de tempo. Pode auxiliar na investigação de distúrbios de aprendizagem associados a períodos de hiperatividade e desatenção na escola. Os registros são obtidos de pacientes em seu ambiente normal (trabalho, escola, residência), em atividades da vida diária, não estando, portanto, limitados à curta duração do registro, nem a ambientes artificiais de laboratórios ou hospitais. A monitorização contínua assegura que distúrbios transitórios da função cerebral, que poderiam não ser detectados no EEG de rotina, sejam documentados e que se estabeleça o diagnóstico diferencial das crises e distribuição das descargas epilépticas por longos períodos.

Na avaliação diagnóstica e acompanhamento evolutivo de pacientes epilépticos é que se encontram os maiores benefícios desse sistema, possibilitando obter dados quantitativos em termos de freqüência das descargas eletrográficas e registro do número de crises clínicas, até mesmo de crises generalizadas de ausências e crises parciais complexas, bem como a sua relação com fatores precipitantes. A MEAP pode detectar anormalidades epileptiformes (ictal e interictal) 10 vezes mais que o EEG convencional (Ebersole, 1993).

Este método pode ser também de importância no diagnóstico diferencial, já que mesmo com uma adequada investigação clínica, associada a métodos de neuro-imagem e eletrencefalografia convencional, a natureza das crises de

Trabalho realizado com apoio financeiro do $\mathrm{CNPq}^{1}$ e CAPES ${ }^{2}$. um paciente pode permanecer incerta, dificultando estabelecer se elas são de origem física ou psíquica, se correspondem a quadros sincopais de origem cárdio-circulatória, crises epilépticas, vertigens paroxísticas, crises conversivas (histéricas), crises de pânico e outras condiçōes paroxísticas bizarras de difícil diagnóstico, que podem até coexistir com verdadeiras crises epilépticas (Ebersole, 1988; 1993). O registro documental dessas condições clínicas transitórias é, muitas vezes, a única maneira segura de diagnóstico, e a monitorização ambulatorial torna factível o registro contínuo até que uma crise espontânea ocorra.

A monitorização eletrencefalográfica pode ser feita também em PS e UTI, quando são internados pacientes com suspeita de crises epilépticas, especialmente durante a noite e fins de semana, ou quando por qualquer razão um EEG convéncional não estiver disponível. Nesses casos pode-se utilizar 4-5 eletrodos auto-aderentes (eletrodos de ECG para recém-nascidos ou eletrodos para condução nervosa) colocados em pele glabra, em montagem abaixo da linha anterior de implantação dos cabelos ("sub-hair line montage") (Bridgers \& Ebersole, 1988; Ebersole \& Bridgers, 1987).

A MEAP pode permitir também uma avaliação objetiva da resposta ao tratamento em relação ao efeito no número e na duração das crises epilépticas, bem como na distribuição circadiana das mesmas. Com essas informações, as doses e horários dos medicamentos podem ser melhor ajustados às necessidades individuais dos pacientes. A monitorização e quantificação das frequiências de alterações eletrencefalográticas, descargas epilépticas e alentecimentos paroxísticos da atividade de base, difusa ou focal, independente do estado de vigília ou sono do paciente, permitem melhor acompanhar a evolução de diversos quadros clínicos durante períodos prolongados de tempo (Blomquist \& Zetterlund, 1985).

\section{Summary}

Recent methods for diagnosis and differential diagnosis of epilepsies are described, mainly structural (CT and MRI) and functional (PET and SPECT) neuroimaging methods, EEG Brain Mapping, Ambulatory EEG Monitoring and video-EEG Recording.

\section{Key-words}

Epilepsies, CT, MRI, PET, SPECT, Brain Mapping, Ambulatory EEG Monitoring, Video/EEG Recording.

\section{Bibliografia}

1 BERCOVIC, S.F; NEWTON, M.R. \& ROWE, C.C. - Localization of Epileptic Foci using SPECT. In Epilepsy Surgery - Lüders, H. New York, 1991, Raven Press Ltd.

2 BLOMQUIST, H.K \& ZETTERLUND, B. - Evaluation of treatment in typical absense seizures: The roles of long-term EEG monitoring and ethosuximide. Acta Pediatrica Scandinavica, 74:409-15, 1985. 
3 BRADY, T.J. \& PYKETT, I.L. - Nuclear Magnetic Ressonance. Semi- , nars in Nuclear Medicine, 13:305-396, 1983.

4 BREITILING, D; GUENTHER, W. \& RONDOT, P - Auditory perception of music measured by brain electrical mapping. Neurophysiologia, 25:765-774, 1987.

5 BROWN, W.S. \& LEHMANN, D. - Verb and non meaning of homophone words activate different cortical generators: a topographical study of evoked potencial fields. Exp. Brain Res. 2 (suppl.):159-68, 1979.

6 CENDES. F.; ANDERMANN,F. - Ressonância Magnética na avaliaçāo das epilepsias: o papel do estudo volumétrico da amígdala e hipocampo. Em Epilepsia - Guerreiro, C.A.M \& Guerreiro, M.M. (Eds.) São Paulo, Lemos Editorial, 1993. pg 135-42.

7 DEVOUS, M.D. Sr.; LEROY, R.F. \& HOMAN, R.W. - Single Photon Emission Computed Tomography in Epilepsy. Seminars in Nuclear Medicine, 20:325-41, 1990.

8 EBERSOLE, J.S. \& BRIDGERS, S.L. - Cassete EEG monitoring in the emergency room and intensive care unit. J. Clin. Neurophysiol. 4:213,1987.

9 EBERSOLE, J.S. (Ed.) - Ambulatory EEG Monitoring. New York, 1988 - Raven Press.

10 EBERSOLE. J.S. - EEG Polygraphic Monitoring - Ambulatory EEG and Polygraphic Monitoring - American Academy of Neurology - Annual Meeting, 1993.

11 ENGEL, J.Jr. - Positron Emission Tomography (PET) in diagnosis of epilepsy. In: Porter, R.J. \& Morselli, P.L. (ed) The Epilepsy. London, England, Butherworth \& Co., 242-66, 1986.

12 ENGEL, J. Jr.; HENRY, TR; RISINGER, M.W. - The role of Positron Emission Tomography in presurgical evaluation of temporal lobe epilepsy. In Lüders, HO Epilepsy Surgery. New York, Raven Press, 231-50. 1992.

13 FREEMAN, W.J. \& MAURER, K. - Images and imaginings from computerized brains. Psychiatr. Res. 29:234-45, 1989.

14 GOTMAN, J. - Pratical use of computer-assisted EEG interpretation in Epilepsy. J. Clin. Neurophysiol. 2:251-65, 1985.

15 GREGORY, D.L. \& WONG, P.K.H - Clinical Relevance of dipole field in rolandic spikes. Epilepsia, 33(1):36-44, 1992.

16 HOLMAN, B.L. \& DEVOUS, M.D. Sr. - Functional Brain SPECT: The emergence of a powerful clinical method. J. Nucl. Med., 33:1888-904, 1992.

17 HUGHES, JR.; TABER, J.E. \& FINO, J.J. - The effect of spikes and spike-free epochs on topographic brain maps. Clin. Eletroencephalogr., 22 (93): 150-160, 1991.

18 IVES, J.R. \& WOODS, J.F. - 4-chanel 24-hour cassete recorder for long-term EEG monitoring of ambulatory pacients. Eletroenceph. Clin. Neurophisiol. 39:88-92, 1975

19 IVES, J.R.; N.R. MAINWARING; D.L.O. SCHOMER - Monitoraçāo Eletroencefalográfica (EEG). Em Epilepsia (Cap. V) C.A.M. Guerreiro \& M.M. Gurereiro (Eds.) São Paulo, Lemos Editorial - 1993, pg. 39-52.
20-JACK, C.R.; SHAREBROUGH, F.W.; TWOMEY, CKET AL. - Temporal lobe seizures: lateralization with MR volume mesurements of the hippocampal formation. Radiology, 175:423-29, 1990.

21 JACOBS, E.C.; BURGERS, R.C.; COLLURA, T.F. - Display and Hardcopy Technology of EEG signal - In:Epilepsy Surgery (Cap 38) Lüders, H. New York, Raven Press Ltd, 1991.

22 JEFFREY, P.J.; MONSEIN, L.H.; SZABO, Z.; HART J.; FISHER, R.P.; LESSER, R.S.; DEBRUN, G.; GORDON, B.; WAGNER, H.N. \& CAMARGO, E.E. - Mapping the distribution of amytal in the intra-arterial sodium amytal (WADA) test with Tc-99m-HMPAO SPECT. Radiology, 178:847-850, 1991.

23 LOMBROSO, C.T. \& DUFFY, F.H. - Brain electrical acitivity mapping in the epilepsies. In: Akimoto, $\mathrm{H}$ \& Kazamatsuti, $\mathrm{H}$ (Eds), Advances in Epileptology, New York, Raven Press, 1982.

24 MAINWARING, N.R.; IVES, J.R.; COSGROVE, G.R.; BLUME, H.W.; SCHOMER, D.L. - High resolution EEG output using a laser print. Electroencephalogr. Clin. Neurophysiol. 79:73-7, 1991.

25 MAURER, K. \& DIERKS, T. - Atlas of Brain Mapping. Springer-Verlag, 1991.

26 MIYAUCHI, T.; ENDO, K.; YAMAGUCHI, T.; HAGIMOTO, H. - Computerized analysis of EEG background activity in epileptic patients, Epilepsia, 32(96):870-881, 1991.

27 NAKAMURA, K.;TUKATANI, Y.; KUBO, A.; HASHIMOTO, S.; TERAYAMA, Y.; AMANO, T. - The behavior of $99 \mathrm{mT}$ c-hexamethylpropyleneamineoxime (99mTc-HMPAO) in blood and brain. Eur. J. Nucl. Med., 15:1000-1007, 1989.

28 PETTSCHE, T.T.; POCKBERGER, H.; RAPPELBERGER, P. - EEG Topography and Mental Performance. In Topography Mapping of Brain Electrical Activity. Duffy, CH (Ed.), Boston, Butterworths, 1986, pg. 63-98.

29 PANET, DR \& GOTMAN, J. - Asymmetry in delta activity in patients with focal epilepsy - Electroencephalogr. Clin. Neurophysiol. 75:474-81, 1990.

30 PETROFF, O.A.C.; PRITCHARD, J.W.; BEHAR, K.L.; ALGER, J.R.; SHULMAN, R.R. - In vivo phosphorus nuclear magnetic resonance spectroscopy in status epilepticus. Annals of Neurology, 16:169-177, 1984.

31 RAUSCH, R. \& LANGFITT, J.T. - Memory Evaluation During the Intracarotid Sodium Amobarbital Procedure, Cap.58. In Epilepsy Surgery, HO Lüders (Ed.), New.York, Raven Press, 1991, pg 507-514.

32 VUONG, T.A. \& BURGEN, R.C. - Split-Screen Video/EEG Reconding versus Synchronized but separate video and EEG acquisition. Chap. 32. In Epilepsy Surgery. H. Lüders (Ed.) New York, Raven Press, 1991.

33 WIESER, H.G. - Pressurgical Evaluation in Adults. - Annual Course American Academy of Neurology. Course No. 125 (Dir. H.O. Lüders), 1993. 